\title{
Aprendizaje basado en problemas en la formación de estudiantes de enfermería. Impacto en la práctica clínica
}

\author{
M. Teresa Alcolea-Cosín, Cristina Oter-Quintana, Rosa M. Martínez-Ortega, Tomás Sebastián-Viana, \\ Azucena Pedraz-Marcos
}

Objetivo. Evaluar el impacto en la práctica clínica de la implantación de la metodología del aprendizaje basado en problemas (ABP) aplicada a los estudios de enfermería.

Sujetos y métodos. Estudio longitudinal prospectivo en dos cohortes de estudiantes de enfermería: el grupo intervención fue la promoción 2005-2006 de la escuela de enfermería, que recibió su educación con la metodología del ABP, y el grupo control fue la promoción de 2004-2005, que recibió su formación a través de otras metodologías docentes, fundamentalmente clases magistrales y seminarios. Se analizó la posible asociación, en cada uno de los elementos del formulario de evaluación del tutor, entre la calificación obtenida por los estudiantes y la metodología de aprendizaje empleada durante su educación (ABP o no ABP). Los criterios de evaluación fueron: responsabilidad, iniciativa, habilidad y destreza en técnicas y procedimientos manuales, organización y metodología en las actividades, comunicación/relación con usuarios y familiares y comunicación/relación con el equipo de trabajo, y grado de consecución de los objetivos docentes.

Resultados. Se obtuvieron 651 formularios de evaluación válidos, 383 para el grupo control y 268 para el grupo intervención. Se encontraron diferencias estadísticamente significativas sólo en el tercer año, en la categoría 'habilidad y destreza en las técnicas relacionadas con los procedimientos manuales' ( $p=0,007)$. El grupo control obtuvo una mejor puntuación.

Conclusiones. Los resultados sugieren que el uso de ABP en la formación académica de los estudiantes de enfermería no modifica la puntuación obtenida en su práctica clínica en las categorías recogidas en nuestra herramienta de evaluación.

Palabras clave. Aprendizaje basado en problemas. Enfermería. Evaluación. Práctica clínica.

\section{Assessing the impact on clinical internship of problem-based learning applied to nursing studies}

Aim. To assess the impact on clinical internship of problem-based learning (PBL) methodology applied to nursing studies.

Subjects and methods. Longitudinal and prospective study of two cohorts of nursing students: the intervention group was the promotion 2005-2006 of the nursing school, that received their education with PBL, and the control group was the promotion 2004-2005 of the nursing school, that received their education with other pedagogic methods, mainly lectures and seminars. We analysed the possible correlation in each of the items on the mentor's assessment form between the grade obtained by students and the learning methodology employed during their education (PBL or non-PBL). The assessment criteria were: responsibility; initiative; skill and proficiency in techniques related to manual procedures; organization and methodology in activities carried out; communication/relationship with patients and families; communication/ relationship with the work team; and degree to which training objectives were reached.

Results. We had 651 valid assessment forms, 383 for the control group and 268 for the intervention group. Clear significant differences were found only in the third year, in the 'skill and proficiency in techniques related to manual process' category ( $p=0.007)$. The control group had higher scores.

Conclusions. The results suggest that the use of PBL in students' academic coursework does not modify the score obtained in their clinical internship on the items specified in our assessment tool.

Key words. Assessment. Clinical internship. Nursing. Problem-based learning.
Escuela Universitaria de Enfermería de la Comunidad de Madrid; Leganés, Madrid (M.T. Alcolea-Cosín, C. Oter-Quintana, R.M. MartínezOrtega, A. Pedraz-Marcos). Hospital Universitario de Fuenlabrada; Fuenlabrada, Madrid, España (T. Sebastián-Viana)

Correspondencia: Dra. Azucena Pedraz Marcos. Escuela Universitaria de Enfermería de la Comunidad de Madrid. Avda. Orellana, s/n. Recinto Hospital Universitario Severo Ochoa. E-28911 Leganés (Madrid). Fax: +34916938813

E-mail:

azucena.pedraz@uam.es

Financiación:

Fondo de Investigaciones Sanitarias del Ministerio de Sanidad y Consumo (proyecto PI052806).

Conflicto de intereses: No declarado.

Competing interests: None declared.

Agradecimientos: Al Prof. A. Franco, por su inestimable ayuda en esta investigación.

Resultados parciales de esta investigación fueron presentados en el XVIII Congreso de la Sociedad Española de Educación Médica y publicados como comunicación en Educ Med 2007; 10: 167.

(c) 2012 Educación Médica 


\section{Introducción}

La evolución de las metodologías docentes en la educación superior en las últimas décadas ha sido constante, a partir de la reflexión sobre la necesidad de capacitar a los egresados para abordar la vida profesional con un rango de competencias suficientemente amplio y que abarque los pilares de la educación -aprender a conocer, aprender a hacer, aprender a vivir juntos y aprender a ser- que indica el Informe de la Comisión Internacional sobre la Educación para el siglo XXI [1]. En este marco, la introducción de metodologías en las que el estudiante adquiere un rol participativo y reflexivo de su propia formación ha sido una constante en los currículos de las titulaciones de ciencias de la salud. La evaluación de estas experiencias desde los resultados del aprendizaje y desde las preferencias del estudiante ha venido a confirmar la pertinencia de estos abordajes metodológicos en la formación $[2,3]$.

El aprendizaje basado en problemas (ABP) es una metodología que se ajusta perfectamente a las evoluciones de los sistemas de enseñanza superior porque facilita no sólo la adquisición de conocimiento, sino de otras capacidades como trabajo en equipo, habilidades de comunicación, responsabilidad en el aprendizaje independiente, cooperación en la búsqueda de información, evaluación crítica de la información, escucha activa y respeto de los puntos de vista del otro [4].

Existen numerosos estudios sobre los resultados en adquisición de competencias cognitivas de los estudiantes formados con ABP. En una revisión sistemática que exploraba la influencia del ABP en la adquisición de competencias en los estudiantes de medicina, se encontraron evidencias fuertes del efecto positivo de esta metodología en las dimensiones cognitiva (sobre todo en el manejo del conocimiento) y social [5]. Las revisiones de la literatura científica llevadas a cabo por Albanese y Mitchell [6] o por Smits et al [7] concluyeron que, aunque existe un mayor entusiasmo e implicación de los estudiantes durante el aprendizaje, no se detectaban diferencias en el nivel de conocimientos adquiridos.

En la formación enfermera son escasos los estudios que valoran el ABP como metodología de aprendizaje-enseñanza. Baker encuentra diferencias significativas en el aprendizaje con metodología ABP en competencias relacionadas con liderazgo, relación de ayuda, conocimiento, búsqueda de información e iniciativa, en un estudio realizado a enfermeras del posgrado de Administración en la Universidad de Indiana [8]. Aún son más escasos los estudios que evalúan la eficacia de esta metodo-
Tabla I. Variables sociodemograficas.

\begin{tabular}{lccc}
\hline & Grupo intervención & Grupo control & $p^{\text {a }}$ \\
\hline Sexo & & & \\
\multicolumn{1}{c}{ Hombre } & $7(15,9 \%)$ & $6(9,4 \%)$ & \\
Mujer & $37(84,1 \%)$ & $58(90,6 \%)$ & \\
\hline $\begin{array}{l}\text { Edad } \\
\text { (media) }\end{array}$ & (IC 95\% $=18,88-20,63)$ & $($ IC $95 \%=19,27-21,27)$ & 0,255 \\
\hline
\end{tabular}

a Estadísticos utilizados: chi al cuadrado en sexo y $t$ de Student en edad.

logía en el entorno clínico. El reciente estudio de Wong et al [9], de la Universidad Politécnica de Hong Kong, sobre el ABP en las prácticas de simulación clínica, muestra como hallazgos una mayor capacitación en los estudiantes sometidos a esta metodología en los cuidados centrados en el paciente, el aprendizaje autodirigido, el aprendizaje inductivo y la transformación del conocimiento teórico en información práctica.

Uys et al [10] han estudiado el impacto del ABP en la práctica asistencial, comparando dos poblaciones de enfermeras, egresadas hacía 6-9 meses, cuya diferencia estribaba en si éstas habían cursado su formación en universidades que tenían o no implementada dicha metodología en el currículo formativo. Siguiendo la clasificación de competencias propuesta por Benner [11], quien señala cinco niveles competenciales - principiante, principiante avanzada, competente, hábil y experta-, se llegó a la conclusión de que las enfermeras formadas en el currículo tradicional alcanzaban en su mayoría el grado de competentes, mientras que las formadas mediante ABP llegaban al nivel de hábil.

La Escuela Universitaria de Enfermería de la Comunidad de Madrid, adscrita a la Universidad Autónoma de Madrid, ha llevado a cabo un proyecto de innovación docente consistente en la aplicación del ABP de manera transversal en el currículo de los alumnos que acceden al centro desde el curso 2005-2006 [12]. En su conjunto, este proyecto está inserto en una corriente de evolución de la educación enfermera hacia un aprendizaje autodirigido y continuado.

La escasez en el número de experiencias de aplicación del ABP de manera transversal en España en la formación enfermera y la ausencia de evaluaciones publicadas al respecto, nos impulsó, a finales del año 2004, a llevar a cabo un estudio cuyo objetivo ha 
Tabla II. Resultados globales de las calificaciones obtenidas en evaluaciones de prácticas clínicas.

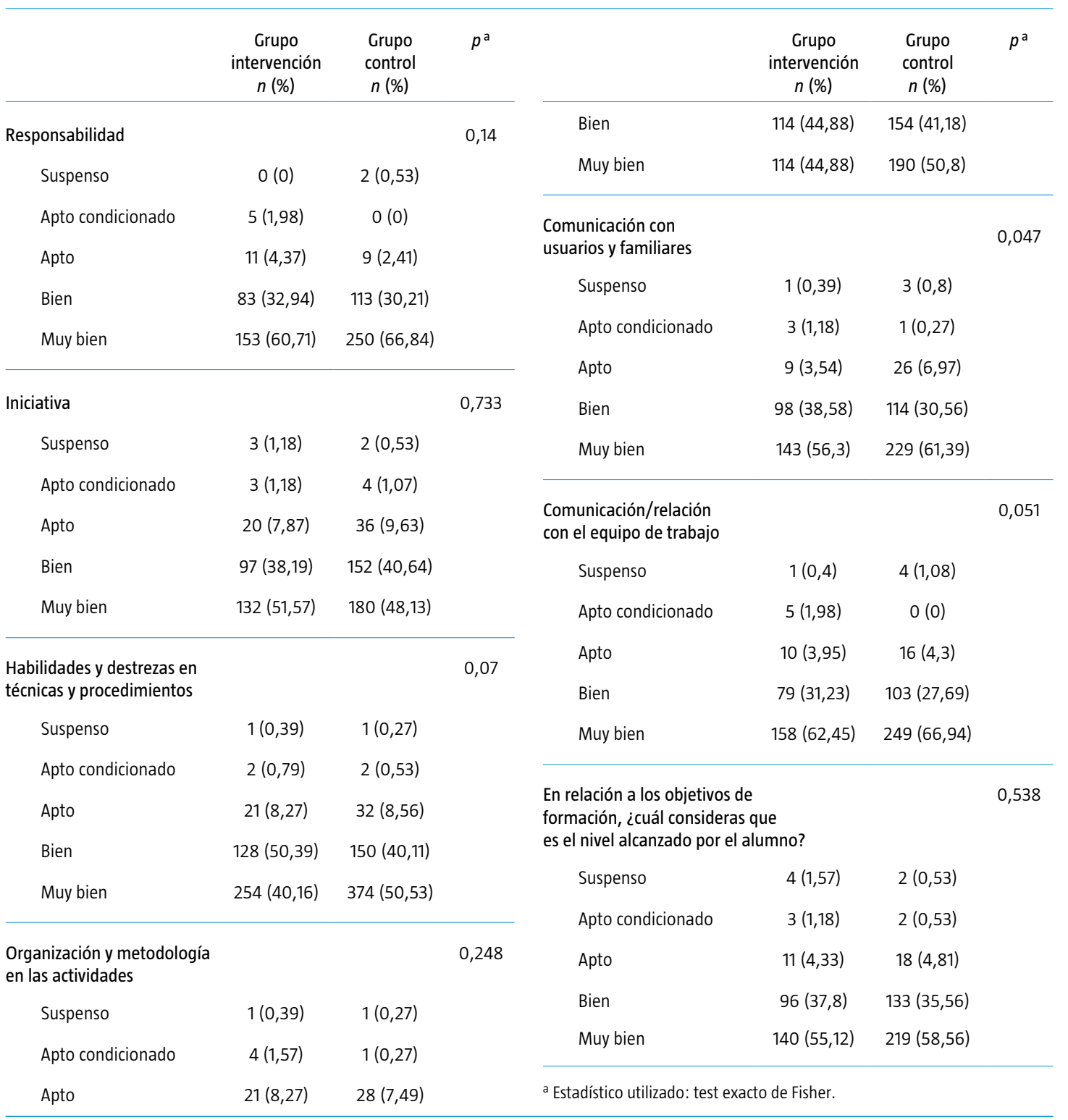

sido medir el impacto de la metodología ABP sobre la práctica clínica de los estudiantes de enfermería.

\section{Sujetos y métodos}

Estudio longitudinal prospectivo en dos cohortes de estudiantes de enfermería. La población de estudio quedó constituida por:
- Grupo intervención: todos los estudiantes que iniciaron la titulación de Enfermería, en el curso 2005/2006, en la Escuela Universitaria de Enfermería de la Comunidad de Madrid, y que han recibido formación con metodología ABP.

- Grupo control: todos los estudiantes que iniciaron la titulación de Enfermería, en el curso 2004/2005, en la Escuela Universitaria de Enfermería de la Comunidad de Madrid, y que reci- 
Tabla III. Resultados de las calificaciones obtenidas en evaluaciones de prácticas clínicas: primer curso.

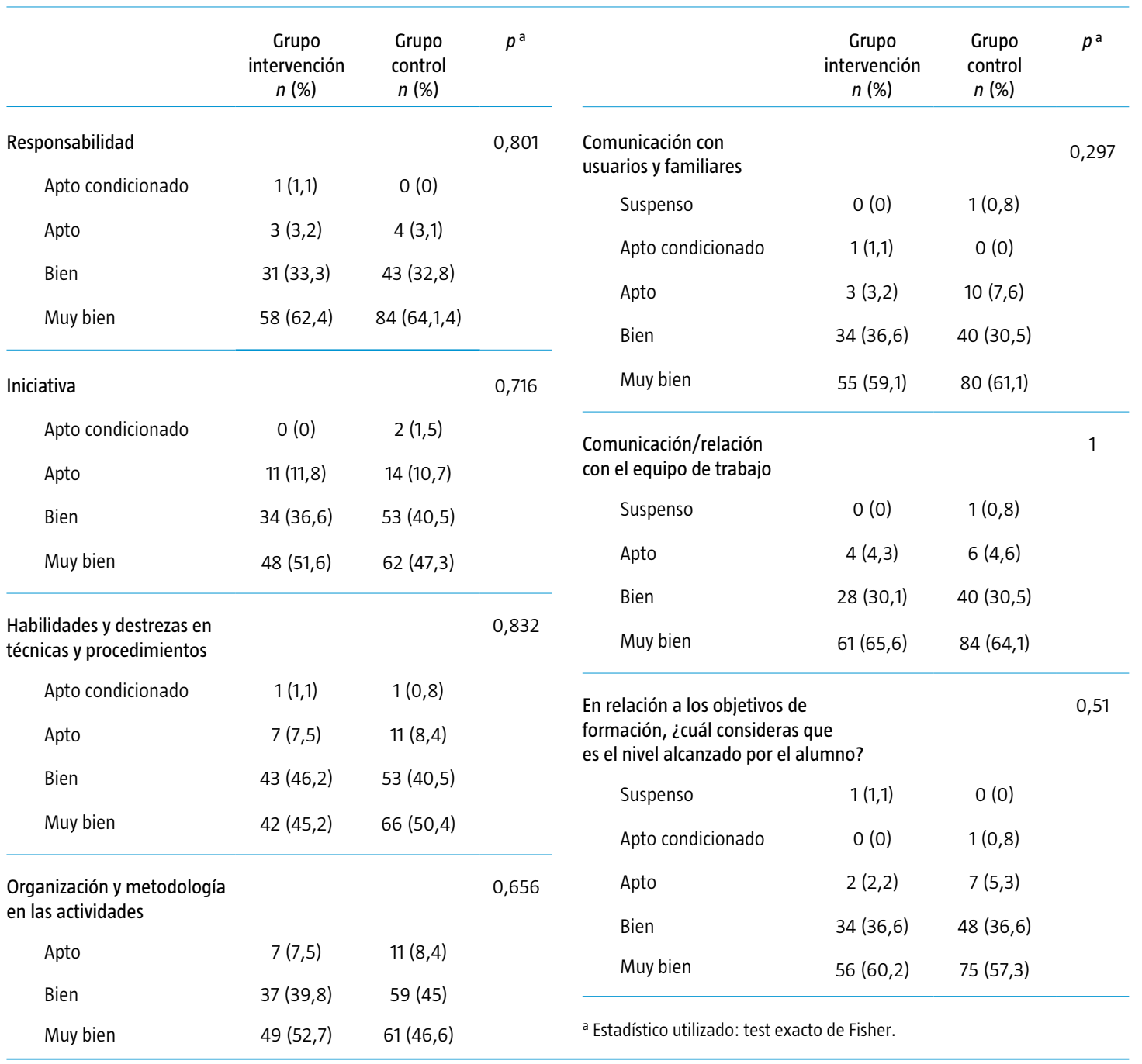

bieron formación fundamentalmente mediante clases magistrales y seminarios.

Se excluyeron del estudio aquellos estudiantes que repitieron curso, abandonaron sus estudios o se incorporaron al grupo intervención en segundo o tercer curso de la titulación de Enfermería en la Escuela Universitaria de Enfermería de la Comunidad de Madrid

\section{Variables}

- Independiente: metodología docente, contemplándose dos categorías, formación con metodología
ABP (grupo intervención) y formación no ABP (grupo control).

- Dependiente: calificación obtenida por el estudiante en las siete categorías de evaluación (responsabilidad, iniciativa, habilidad y destreza en técnicas y procedimientos manuales, organización y metodología en las actividades, comunicación/relación con usuarios y familiares y comunicación/relación con el equipo de trabajo) que recoge el documento cumplimentado por el tutor de la práctica clínica durante los seis períodos de prácticas que desarrolla el estudiante en su formación de pregrado. Cada categoría de evaluación se organiza en una escala que contempla las 
Tabla IV. Resultados de las calificaciones obtenidas en evaluaciones de prácticas clínicas: segundo curso.

\begin{tabular}{|c|c|c|c|c|c|c|c|}
\hline & $\begin{array}{c}\text { Grupo } \\
\text { intervención } \\
n(\%)\end{array}$ & $\begin{array}{c}\text { Grupo } \\
\text { control } \\
n(\%)\end{array}$ & $p^{a}$ & & $\begin{array}{c}\text { Grupo } \\
\text { intervención } \\
n(\%)\end{array}$ & $\begin{array}{l}\text { Grupo } \\
\text { control } \\
n(\%)\end{array}$ & $p^{a}$ \\
\hline Responsabilidad & & & 0,046 & Bien & $29(39,7)$ & $44(35,2)$ & \\
\hline Suspenso & $0(0)$ & $2(1,6)$ & & Muy bien & $36(49,3)$ & $71(56,8)$ & \\
\hline Apto condicionado & $3(4,2)$ & $0(0)$ & & & & & \\
\hline Apto & $3(4,2)$ & $2(1,6)$ & & $\begin{array}{l}\text { Comunicación con } \\
\text { usuarios y familiares }\end{array}$ & & & 0,834 \\
\hline Bien & $25(3,2)$ & $36(28,8)$ & & Suspenso & $1(1,4)$ & $1(, 8)$ & \\
\hline Muy bien & $40(56,3)$ & $85(68)$ & & Apto condicionado & $1(1,4)$ & $1(0,8)$ & \\
\hline & & & & Apto & $2(2,7)$ & $7(5,6)$ & \\
\hline IIIriaciva & & & 0,158 & Bien & $26(35,6)$ & $40(32,3)$ & \\
\hline Suspenso & $0(0)$ & $2(1,6)$ & & Muy bien & $43(58,9)$ & $75(60,5)$ & \\
\hline Apto condicionado & $2(2,7)$ & $0(0)$ & & & & & \\
\hline Apto & $8(11)$ & $11(8,8)$ & & $\begin{array}{l}\text { Comunicación/relación } \\
\text { con el equipo de trabajo }\end{array}$ & & & 0,185 \\
\hline Bien & $23(31,5)$ & $53(42,4)$ & & Suspenso & $0(0)$ & $2(1,6)$ & \\
\hline Muy bien & $40(54,8)$ & $59(47,2)$ & & Apto condicionado & $3(4,2)$ & $0(0)$ & \\
\hline Habilidades y destrezas en & & & 0,686 & Apto & $2(2,8)$ & $4((3,3)$ & \\
\hline & & & & Bien & $20(27,8)$ & $31(25,2)$ & \\
\hline Suspenso & $0(0)$ & $1(0,8)$ & & Muy bien & $47(65,3)$ & $86(69,9)$ & \\
\hline Apto condicionado & $1(1,4)$ & $0(0)$ & & & & & \\
\hline Apto & $6(8,2)$ & $10(8,0)$ & & \multirow{2}{*}{\multicolumn{2}{|c|}{$\begin{array}{l}\text { En relación a los objetivos de } \\
\text { formación, icuál consideras que } \\
\text { es el nivel alcanzado por el alumno? }\end{array}$}} & & 0,133 \\
\hline Bien & $34(46,6)$ & $53(42,4)$ & & & & & \\
\hline Muy bien & $32(43,8)$ & $61(48,8)$ & & Suspenso & $0(0)$ & $2(1,6)$ & \\
\hline Organización y metodología & & & 0,641 & Apto condicionado & $3(4,1)$ & $0(0)$ & \\
\hline en las actividades & & & & Apto & $5(6,8)$ & $5(4)$ & \\
\hline Suspenso & $0(0)$ & $1(0,8)$ & & Bien & $26(35,6)$ & $44(35,2)$ & \\
\hline Apto condicionado & $2(2,7)$ & $1(0,8)$ & & Muy bien & $39(53,4)$ & $74(59,2)$ & \\
\hline Apto & $6(8,2)$ & $8(6,4)$ & & \multicolumn{3}{|c|}{ a Estadístico utilizado: test exacto de Fisher. } & \\
\hline
\end{tabular}

puntuaciones 'muy bien', 'bien', 'apto, 'apto condicionado' y 'suspenso.' Esta herramienta de evaluación fue elaborada por un grupo de expertos hace 15 años, con una estructura similar a la utilizada en otras escuelas universitarias de enfermería españolas. Todos los tutores que evaluaron a los estudiantes participantes en el estudio recibieron instrucciones por escrito acerca de la cumplimentación de la herramienta de evaluación con respecto a las categorías contempladas en ella y el significado de las calificaciones dadas.

\section{Recogida y análisis de datos}

Se ha realizado un análisis descriptivo de las calificaciones obtenidas en la práctica clínica por los estudiantes que iniciaron sus estudios en los cursos académicos 2004/2005 (grupo control) y 2005/2006 
Tabla V. Resultados de las calificaciones obtenidas en evaluaciones de prácticas clínicas: tercer curso.

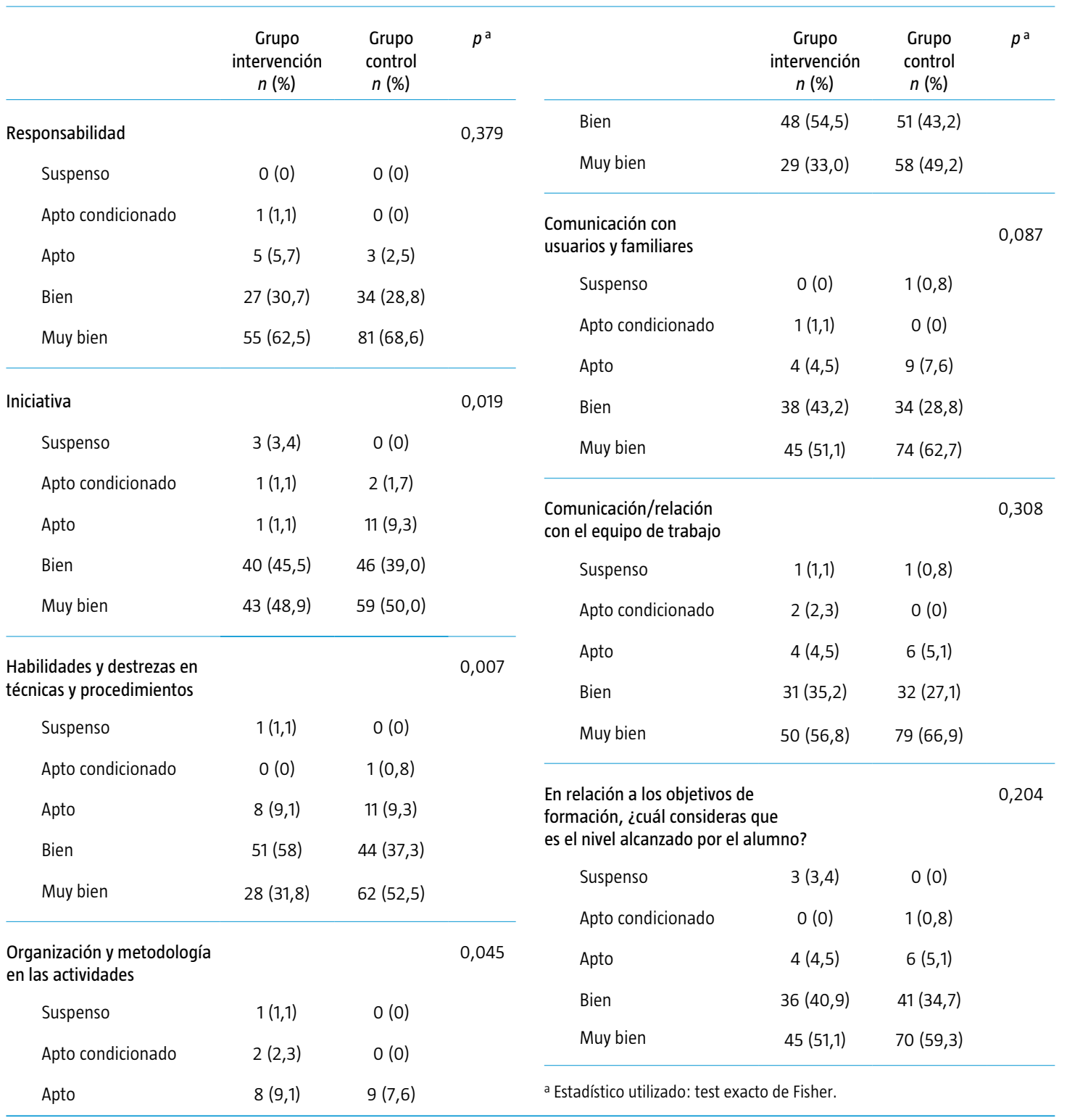

(grupo intervención) durante los tres años de duración de la formación conducente a la obtención del título de Diplomado en Enfermería. Se han realizado dos mediciones por curso académico, cada una de ellas correspondiente a un período de práctica clínica. Se ha analizado la posible asociación en cada una de las categorías que recoge la evaluación de la formación práctica entre la calificación dada por el tutor de la práctica clínica (muy bien, bien, apto, apto condicionado y suspenso) y la metodología de aprendizaje empleada (ABP, no $\mathrm{ABP})$, usando el test de chi al cuadrado o el test exacto de Fisher en el caso de que más del $20 \%$ de las celdas tuvieran valores esperados menores de 5 . Se ha comprobado la distribución de las variables frente a los modelos teóricos. En todos los contrastes de hipótesis se ha rechazado la hipótesis nula con un error de tipo I o error $\alpha<0,05$. 


\section{Resultados}

El grupo intervención estuvo constituido por 49 estudiantes, que corresponde al número de alumnos que iniciaron sus estudios en la titulación universitaria de Enfermería en el año 2005-2006. El grupo control estuvo conformado por los 68 estudiantes que iniciaron la diplomatura el año anterior.

Las características sociodemográficas respecto a las variables de edad y sexo de los estudiantes del grupo intervención y control pueden observarse en la tabla I. No se han encontrado diferencias estadísticamente significativas entre ambos grupos en la distribución por sexos ni en la edad de los alumnos.

En cuanto a los estudios previos que los estudiantes de ambos grupos habían realizado antes de su ingreso en la universidad para desarrollar sus estudios de la Diplomatura en Enfermería, la mayoría procedían de bachillerato $(83,5 \%$ en el grupo intervención y $85,2 \%$ en el grupo control), siendo también homogéneos los grupos en cuanto a su formación previa.

En el periodo de estudio se recogieron un total de 654 documentos de evaluación, de los cuales fueron válidos para el análisis 628 documentos. De ellos, 374 correspondían al grupo control y 254 al grupo intervención. En la tabla II se presentan los resultados del análisis conjunto de las calificaciones obtenidas por los estudiantes en cada uno de las variables, agrupadas en función de su pertenencia al grupo intervención o control.

En la tabla III se presentan las calificaciones obtenidas en las evaluaciones de las dos rotaciones clínicas de los alumnos de primer curso. De este curso se recogieron 224 evaluaciones, de las cuales 93 correspondían al grupo intervención y 131 al grupo control. En la tabla IV se presentan los resultados obtenidos por los alumnos de segundo curso; en total se recogieron 198 evaluaciones, de las cuales 73 eran del grupo intervención y 125 del grupo control. Por último, en la tabla $\mathrm{V}$ se presentan los resultados obtenidos por los alumnos de tercer curso; se recogieron un total de 206 evaluaciones, de las cuales 88 eran del grupo intervención y 118 del grupo control.

De los resultados destaca que en la evaluación global y en la de los alumnos de primero y de segundo curso, las puntuaciones son similares, aunque con mayor porcentaje de evaluados como 'muy bien' en el grupo control, excepto en la iniciativa, que es mayor la evaluación 'muy bien' en el grupo intervención; sin embargo, ninguna de las diferencias encontradas es estadísticamente significativa.

En el análisis de las evaluaciones de tercer curso aparecen diferencias que tienen mayor significación estadística; destaca especialmente las habilidades y destrezas $(p=0,007)$, presentando diferencias más claras las puntuaciones de los dos grupos. El grupo control presenta mayores porcentajes en todas las variables de la evaluación 'muy bien', mientras que en el grupo intervención la evaluación que predomina en las variables de estudio es 'bien'.

\section{Discusión}

Como se señala en la reciente revisión sistemática llevada a cabo por Murad y Varkey [13], existe escasa evidencia del impacto de metodologías de aprendizaje autodirigido en los resultados de los estudiantes de ciencias de la salud. Entre los 106 artículos revisados, existían pocos estudios controlados y aleatorizados, siendo la mayoría de orden descriptivo. Entre las recomendaciones de estos autores se plantea la necesidad de llevar a cabo investigaciones de mayor nivel de evidencia, que contemplen también algunas características específicas de los estudiantes (como nivel de entrenamiento, experiencias prácticas previas...). En este sentido, nuestra investigación se encuentra con las mismas limitaciones metodológicas que describen los autores señalados, en relación con la dificultad en la aleatorización y control de las muestras.

Por otro lado, y en relación con los escasos estudios existentes acerca del impacto del ABP en la adquisición de competencias profesionales, nuestro estudio sólo encuentra diferencias significativas, a favor del grupo control, en la categoría 'habilidad y destreza en técnicas y procedimientos'. Es posible que la propia herramienta usada en la evaluación de la práctica clínica condicione las diferencias en este sentido, dado que las categorías usadas en ella se sitúan en la esfera de las habilidades y actitudes. El documento de evaluación de la práctica clínica a cumplimentar por el tutor se diseñó previamente a la introducción de los cambios metodológicos en nuestra institución. A la vista de estos resultados, cabría plantearse como una de las limitaciones fundamentales de nuestro estudio la sensibilidad del instrumento utilizado para medir la influencia del $\mathrm{ABP}$ en la formación práctico-clínica de los estudiantes de enfermería.

En conclusión, podríamos destacar que la adquisición de competencias en la formación prácticoclínica de los estudiantes de enfermería no parece estar afectada por el uso de diferentes metodologías de aprendizaje en la formación teórica.

Será necesario revisar el instrumento de evaluación para que incorpore otras competencias que, 
según la bibliografía, pueden verse afectadas por la metodología de ABP, como la flexibilidad, adaptabilidad o capacidad de resolución de problemas, y será preciso realizar nuevos estudios con otros instrumentos con mayor sensibilidad para determinar si la utilización del ABP en la formación teórica de los alumnos tiene impacto en las competencias que éstos desarrollan y aplican en las prácticas clínicas y en su ejercicio profesional posterior.

Bibliografía

1. Delors J. La educación encierra un tesoro: informe de la Unesco de la Comisión Internacional sobre la Educación para el siglo XXI. Madrid: Santillana; 1999.

2. Canalejas-Pérez C, Martínez-Martín ML, Pineda-Ginés C, Vera-Cortés ML, Soto-González M, Martín-Marino A, et al. Estilos de aprendizaje en los estudiantes de enfermería. Educ Med 2005; 8: 83-90.

3. Rassool GH, Rawaf S. Learning style preferences of undergraduate nursing students. Nurs Stand 2007; 21: 35-41.

4. Wood D. ABC of learning and teaching in medicine. Problem based learning. BMJ 2003; 326: 328-30.

5. Koh GCH, Khoo HE, Wong ML, Koh D. The effects of problem-based learning during medical school on physician competency: a systematic review. Can Med Assoc J 2008; 178: 34-41.

6. Albanese M, Mitchell S. Problem-based learning: a review of literature on its outcomes and implementation issues. Acad Med 1993; 68: 52-81.

7. Smits P, Verbeek J, Buisonjé C. Learning in practice. Problem based learning in continuing medical education: a review of controlled evaluation studies. BMJ 2002; 324: 153-6.

8. Baker CM, McDaniel AM, Pesut DJ, Fisher ML. Learning skills profiles of master's students in nursing administration: assessing the impact of problem-based learning. Nurs Educ Perspect 2007; 28: 190-5.

9. Wong FK, Cheung S, Chung L, Chan K, Chan A, To T, et al. Framework for adopting a problem-based learning approach in a simulated clinical setting. J Nurs Educ 2008; 47: 508-14.

10. Uys L, Gwele N, McInerney P, Van Rhyn L, Tanga T. The competence of nursing graduates from problem-based programs in South Africa. J Nurs Educ 2004; 43: 352-61.

11. Benner P. From novice to expert. Menlo Park, CA: AddisonWesley; 1984.

12. Pedraz-Marcos A, García-González A, Rubiales-Paredes MD Alcolea-Cosín MT, Oter-Quintana C, Palmar-Santos AM, et al. Innovación docente en enfermería: el trabajo del estudiante en el aprendizaje basado en problemas. In: El proceso de aprendizaje en la Universidad Autónoma de Madrid: la implantación del crédito europeo. Madrid: UAM Ediciones; 2008. p. 293-314.

13. Murad MH, Varkey P. Self-directed learning in health professions education. Ann Acad Med Singapore 2008; 37: 580-90. 